\title{
Population-level impact of infant 10-valent pneumococcal conjugate vaccination on adult pneumonia hospitalisations in Finland
}

\author{
Omar Okasha, ${ }_{1}$ Hanna Rinta-Kokko, ${ }^{2}$ Arto A Palmu, ${ }^{2}$ Esa Ruokokoski, ${ }^{2}$ Jukka Jokinen, ${ }^{2}$ \\ J Pekka Nuorti ${ }^{1,3}$
}

\begin{abstract}
- Additional material is published online only. To view please visit the journal online (http://dx.doi.org/10.1136/ thoraxjnl-2017-210440).
\end{abstract}

'Department of Epidemiology, Health Sciences, Faculty of Social Sciences, University of Tampere, Tampere, Finland ${ }^{2}$ Department of Public Health Solutions, National Institute for Health and Welfare (THL), Helsinki, Finland

${ }^{3}$ Department of Health Security, National Institute for Health and Welfare (THL), Helsinki, Finland

\section{Correspondence to}

Prof J Pekka Nuorti, Department of Epidemiology, Health Sciences, Faculty of Social Sciences, University of Tampere, Tampere 33100, Finland; Pekka. Nuorti@uta.fi

The main findings were presented in part at the 10th International Symposium on Pneumococci and Pneumococcal Diseases, Glasgow, Scotland, 26-30 June 2016.

Received 25 April 2017 Revised 18 August 2017 Accepted 18 September 2017 Published Online First 7 October 2017

\begin{abstract}
Introduction Limited data are available on populationlevel herd effects of infant 10-valent pneumococcal conjugate vaccine (PCV10) programmes on pneumonia. We assessed national trends in pneumococcal and allcause pneumonia hospitalisations in adults aged $\geq 18$ years, before and after infant PCV10 introduction in 2010.
\end{abstract}

Methods Monthly hospitalisation rates of International Statistical Classification of Diseases, 10th revision (ICD-10)-coded primary discharge diagnoses compatible with pneumonia from 2004-2005 to 2014-2015 were calculated with population denominators from the population register. Trends in pneumonia before and after PCV10 introduction were assessed with interrupted time-series analysis. Rates during the PCV10 period were estimated from adjusted negative binomial regression model and compared with those projected as continuation of the pre-PCV10 trend. All-cause hospitalisations were assessed for control purposes. Results Before PCV10, the all-cause pneumonia rate in adults aged $\geq 18$ years increased annually by $2.4 \%$, followed by a $4.7 \%$ annual decline during the PCV 10 period. In 2014-2015, the overall all-cause pneumonia hospitalisation rate was 109.3/100 000 (95\% Cl 96.5 to 121.9$)$ or $15.4 \%$ lower than the expected rate. $A$ significant $6.7 \%$ decline was seen in persons aged $\geq 65$ years (131.5/100 000), which translates to 1456 fewer pneumonia hospitalisations annually. In comparison, hospitalisations other than pneumonia decreased by $3.5 \%$ annually throughout the entire study period. Conclusion These national data suggest that herd protection from infant PCV10 programme has reversed the increasing trend and substantially decreased allcause pneumonia hospitalisations in adults, particularly the elderly.

\section{INTRODUCTION}

Lower respiratory infections are the fourth common cause of death globally. ${ }^{1}$ Community-acquired pneumonia (CAP) causes significant clinical and economic burden associated with hospitalisations, particularly in the elderly. ${ }^{2}$ Recent estimates of the proportion of CAP that is attributable to Streptococcus pneumoniae (pneumococcus) in adults have ranged from $19 \%$ to $27 \%$. However, because sensitive and specific assays are not routinely used in clinical practice-particularly for non-hospitalised cases-these estimates may

\section{Key messages}

What is the key question?

- In a nationwide, population-based study, we assessed whether vaccinating infants with the 10-valent pneumococcal conjugate vaccine (PCV10) had had an impact on adult pneumonia hospitalisations through herd protection.

What is the bottom line?

- Although there was an increasing trend in rates of pneumonia before PCV10, five years after infant PCV10 introduction, all-cause pneumonia hospitalisations had decreased significantly in all adult age groups, particularly the elderly.

Why read on?

- In high-income countries, the ageing of population and the uncertain cost-effectiveness of preventing adult pneumococcal disease by direct vaccination highlight the public health significance of the pneumococcal conjugate vaccine programme's indirect impact in reducing the burden of adult pneumonia.

be conservative. ${ }^{3}{ }^{4}$ In a recent prospective cohort study of Finnish adults $\geq 65$ years of age, CAP incidence was estimated to be 1050 cases/100 000 person-years; $17 \%$ of cases were due to $S$. pnenmoniae, and $85 \%$ required hospitalisation. ${ }^{5}$ In the USA, the annual incidence of hospitalised CAP in adults $\geq 80$ years of age was estimated to be $>1600$ cases/100 000. ${ }^{6}$

Prelicensure clinical trials suggested a $20 \%-40 \%$ effectiveness of the 7-valent pneumococcal conjugate vaccine (PCV7) against radiologically confirmed pneumonia in children $\leq 5$ years of age. ${ }^{78}$ In persons $\geq 65$ years of age, one dose of the 13-valent pneumococcal conjugate vaccine (PCV13) reduced vaccine-serotype CAP by $45 \%$ in the Netherlands but had little impact on overall pneumonia. ${ }^{9}$ After introduction of PCV7-and subsequently PCV13-in routine infant immunisation programmes, several population-based studies reported reductions in all-cause pneumonia hospitalisations in children. ${ }^{10}{ }^{11}$ In Finland, 10-valent pneumococcal conjugate vaccine (PCV10) introduction was recently shown to have substantially 
decreased the incidence of pneumonia in both vaccine-eligible and older, unvaccinated children. ${ }^{12}$ Infant pneumococcal conjugate vaccines (PCVs) decrease carriage of vaccine-serotype pneumococci and, consequently, transmission to unvaccinated groups. ${ }^{13}$ Few studies, however, have evaluated the population-level herd effects of infant PCVs on adult pneumonia hospitalisations. ${ }^{14-17}$ Given the increasing burden of pneumonia hospitalisations associated with ageing of the population, reducing morbidity and mortality from pneumonia in adults by infant pneumococcal vaccination would yield major public health benefits. ${ }^{18}$

We conducted a nationwide register linkage study to assess the public health impact of infant PCV10 programme introduction on all-cause and pneumococcal pneumonia hospitalisations in adults $\geq 18$ years of age in Finland.

\section{METHODS}

\section{Pneumococcal vaccination in Finland}

In September 2010, PCV10 was introduced in the Finnish National Vaccination Programme (NVP) under a three-dose schedule (at 2, 5 and 12 months of age) without catch-up programme. All children born after June 2010 were eligible. In the 2012 birth cohort, the uptake of at least one dose of PCV10 was estimated to be $94 \% .{ }^{19}$ Use of the 23 -valent pneumococcal polysaccharide vaccine (PPSV23) and PCV13 in adults at risk and the elderly is recommended. However, there is no national adult vaccination programme, and in 2014 the cumulative coverage of both vaccines in adults was $<5 \%$ on the basis of vaccine distribution data.

\section{Study population and data sources}

This was a nationwide, population-based, quasiexperimental study. The national hospital discharge register includes discharge notifications for inpatient admissions and outpatient visits from all Finnish hospitals. International Statistical Classification of Diseases, 10th revision (ICD-10)-coded discharge diagnoses and visit dates for pneumonia hospitalisations in all adults $\geq 18$ years of age from 2004-2005 to 2014-2015 (epidemiological years from July to June) were extracted; population denominators were from the population information system. Data were analysed in the following age groups: $18-49,50-64, \geq 65$ and $\geq 18$ years of age. The age group $\geq 65$ years was further divided into $65-74,75-84$ and $\geq 85$ years of age.

\section{Pneumonia definitions}

All-cause pneumonia hospitalisation was defined as record of a patient hospitalised for at least overnight with ICD-10-coded pneumonia as the primary discharge diagnosis (J10-J18 and J86; online supplementary tables 1 and 2). Pneumococcal pneumonia and empyema were defined as patients with ICD-codes $\mathrm{J} 13$ and J86, respectively, in any discharge diagnosis field, with or without overnight hospitalisation. Potential multiple pneumonia discharge records for the same patient within 90 days from the date of the index pneumonia diagnosis were combined into one episode. The hospital discharge dataset included no radiological data. All-cause hospitalisations, defined as records of patients hospitalised for at least overnight with ICD-10 discharge codes other than J10-J18 and J86, were assessed for comparison.

\section{Statistical analysis}

Interrupted time-series analysis was used to compare rates of adult pneumonia before and after infant PCV10 introduction. The comparison periods for analysis included the pre-PCV10 period (epidemiological years from 2004-2005 to 2009-2010; ie, 72 monthly data points) and the PCV10 period (epidemiological years from 2011-2012 to 2014-2015; ie, 48 monthly data points); 2010-2011 was considered a transitional period and excluded from the analysis.

Separate models were fitted for each case definition (all-cause pneumonia, pneumococcal pneumonia, empyema and all-cause hospitalisations), age group and for the whole adult population. In all models, the outcome was the monthly number of episodes with the $\log$ of the population/100 000 as offset, ${ }^{20}$ and the reported measure was monthly incidence rate ratio (IRR), which was exponentiated to estimate the annual IRR. The model parameters included the baseline rate at the beginning of the study and the trend before and after PCV10 introduction. Our model did not include the change in level as an immediate effect after the intervention because this is more relevant for studying the direct effect of PCVs on pneumonia hospitalisations in vaccine-eligible children. ${ }^{11}$ In contrast, PCV-attributed herd protection in adults appears to have a 'lag period' before coming into full effect ${ }^{21}$ and in the absence of a clear definition of such lag period, setting the time point for the change in level a priori would have been largely subjective. All models were adjusted for sex, and models for the aggregate age groups were age adjusted. To account for seasonal fluctuation in rates, all models included a Fourier seasonality component with the linear combinations of sine and cosine functions:

$$
\begin{aligned}
\log \mathrm{E}\left(\mathrm{Y}_{\mathrm{t}}\right) & =\log \left(\mathrm{C}_{\mathrm{t}}\right)+\beta_{0}+\beta_{1} \mathrm{~T}_{\mathrm{t}}+\beta_{2} \mathrm{X}_{\mathrm{t}} \mathrm{T}_{\mathrm{t}} \\
& +\beta_{3} \sin [2 \pi / 12]+\beta_{4} \cos [2 \pi / 12]+\beta_{5} \mathrm{G}_{\mathrm{t}}
\end{aligned}
$$

where $\mathrm{Y}_{t}$ is the number of pneumonia episodes measured at month $t \cdot \log \left(\mathrm{C}_{\mathrm{t}}\right)$ is the offset equal to the $\log$ of the population $C_{t}$ divided by $100000 . \beta_{0}$ is baseline rate. $T_{t}$ is the time since the beginning of the study until month $t$, and $\beta_{1}$ is the pre-PCV10 slope. $X_{t} T_{t}$ is an interaction term and $\beta_{2}$ represents the post-PCV10 trend. The sine and cosine terms represent the Fourier seasonality component. $G_{t}$ is a binary representing sex.

Trends in pneumonia episodes before and after PCV10 were compared by estimating annual IRRs per 100000 with the corresponding 95\% CI. The IRR of the trend before PCV10 is estimated as the change in annual hospitalisation rates from the first year in the observation period. The IRR of the trend after PCV10 is estimated as the comparison of the annual trend in hospitalisation rate following the start of the PCV10 period to the period before. Percentage annual changes in trend were calculated as $($ IRR -1$) \times 100$.

To quantify the indirect impact of PCV10, the rate at the end of the study period (ie, epidemiological year 2014-2015) was estimated as the non-linear prediction from the model with the full set of parameters and was then compared with the expected rate that would have occurred in the absence of PCV10 introduction. The expected rates were non-linearly predicted as continuation of the trend in the period before PCV10, by holding the model parameter denoting the trend after PCV10 at zero. ${ }^{22}$ The number of prevented pneumonia admissions per year was estimated by multiplying the annual absolute rate reduction by the population size in 2014-2015. To smoothen the seasonal variation in the graphical presentation, symmetrical 12 -month moving average filters were applied to average monthly estimated and expected rates. Incidence rate residual analysis was done, including tests for autocorrelation and partial autocorrelations. These indicated no significant deviances from model assumptions. The level of statistically significant two-tailed $\mathrm{p}$ value was $<0.05$. Stata/SE V.14 was used in statistical analyses. The study protocol was 
Table 1 Hospitalisations for pneumonia episodes in adults $\geq 18$ years of age, Finland, epidemiological years from 2004-2005 to 2014-2015

\begin{tabular}{|c|c|c|c|c|c|c|c|}
\hline \multirow[b]{3}{*}{ Epidemiological years* } & \multirow{3}{*}{$\begin{array}{l}\text { Total } \\
\text { population } \geq 18 \text { years } \\
\text { of age }\end{array}$} & \multicolumn{6}{|c|}{ Pneumonia episodest } \\
\hline & & \multicolumn{2}{|c|}{ All-cause pneumonia hospitalisations } & \multicolumn{2}{|c|}{ Empyema } & \multicolumn{2}{|c|}{ Pneumococcal pneumonia } \\
\hline & & $n$ & $\begin{array}{l}\text { Rate per } 100000 \\
\text { population }\end{array}$ & $n$ & $\begin{array}{l}\text { Rate per } 100000 \\
\text { population }\end{array}$ & $n$ & $\begin{array}{l}\text { Rate per } 100000 \\
\text { population }\end{array}$ \\
\hline 2004-2005 & 4141374 & 20823 & 502.8 & 198 & 4.8 & 377 & 9.1 \\
\hline $2005-2006$ & 4164582 & 20542 & 493.3 & 179 & 4.3 & 338 & 8.1 \\
\hline $2006-2007$ & 4190862 & 20821 & 496.8 & 197 & 4.7 & 402 & 9.6 \\
\hline 2008-2009 & 4248882 & 23640 & 556.4 & 264 & 6.2 & 516 & 12.1 \\
\hline 2009-2010 & 4276986 & 22394 & 523.6 & 264 & 6.2 & 449 & 10.5 \\
\hline 2010-2011 & 4305246 & 26744 & 621.2 & 273 & 6.3 & 437 & 10.2 \\
\hline 2011-2012 & 4333734 & 28609 & 660.2 & 294 & 6.8 & 441 & 10.2 \\
\hline 2012-2013 & 4361280 & 26376 & 604.8 & 291 & 6.7 & 469 & 10.8 \\
\hline
\end{tabular}

*Years runs from July to June.

tPotential multiple pneumonia discharge records for the same patient within 90 days from the date of the index pneumonia diagnosis were combined into one episode.

approved by the institutional review board in the National Institute for Health and Welfare (THL), Finland. Permissions to use the register data for research were obtained from the register controller at THL.

\section{RESULTS}

\section{Characteristics of pneumonia hospitalisations}

During the study period (2004-2004 to 20142015), >21.7 million hospital discharges were recorded in Finnish adults $\geq 18$ years of age; 263382 (1.2\%) were all-cause pneumonia hospitalisation episodes. Pneumococcal pneumonia and empyema accounted for $1.8 \%$ and $1.1 \%$ of pneumonia episodes, respectively (table 1 ). The baseline rate of and number of all-cause pneumonia hospitalisations in 2004-2005 were $502.8 / 100000$ and 20823 , respectively (table 2 ). The baseline rates ranged from 167.9/100 000 in persons 18-49 years of age to $4434.3 / 100000$ in those $\geq 85$ years of age. The overall baseline rates for pneumococcal pneumonia and empyema were low (9.1 and 4.8/100 000, respectively).

\section{Trends in pneumonia rates during the pre-PCV10 period}

From July 2004 to June 2010, the rates of all-cause pneumonia hospitalisations in adults $\geq 18$ years of age increased by $2.4 \%$ annually (IRR 1.024 ; 95\% CI 1.018 to 1.037 ) (table 2). Similarly, rates of both pneumococcal pneumonia and empyema increased annually by $3.7 \%$ and $6.2 \%$ (IRRs 1.037 and 1.062 , respectively). Age-stratified trend analysis showed increases in rates of all-cause pneumonia hospitalisations during the pre-PCV10 period in all age groups (table 2).

\section{Trends in pneumonia rates during the PCV10 period}

From July 2011 to June 2015, the all-cause pneumonia rates in adults $\geq 18$ years of age decreased annually by $4.7 \%$ (IRR $0.953 ; 95 \%$ CI 0.942 to 0.965 ) (table 2). Statistically significant declines were seen in all age-specific rates except for adults $\geq 75$ years of age; the largest reduction was seen in age groups 18-49 and 50-64 years (9.2\%). The rate of pneumococcal pneumonia decreased annually by $8.1 \%$ (IRR $0.919 ; 95 \%$ CI 0.876 to 0.965 ); significant annual declines of $14.5 \%$ were seen in adults $<65$ years of age. Reductions in empyema rates were non-significant (table 2).

\section{Potential outcomes analysis}

In epidemiological year 2014-2015, the estimated annual rate of all-cause pneumonia hospitalisations in adults $\geq 18$ years of age was 109.3 episodes/100 000 (95\% CI 96.5 to 121.9 ) or $15.4 \%$ lower compared with the expected rate on the basis of pre-PCV10 trends (table 3, figure 1). For pneumococcal pneumonia, the overall rate reduction in 2014 was estimated to be 3.5 episodes per 100000 (95\% CI 2.5 to 4.4 ) or $26.5 \%$. By 2014-2015, the overall reduction in empyema was 1.5 episodes/100 000 (95\% CI 0.9 to 2.1) (table 3).

In age-stratified analyses, statistically significant reductions in all-cause pneumonia hospitalisations were seen in all age groups (table 3, figure 2). Compared with the expected rate, the estimated reductions in all-cause pneumonia hospitalisations in age groups $18-49,50-64$ and $\geq 65$ years were 44.1 (26.9\%), $140.6(28.8 \%)$ and $131.5(6.7 \%)$ per 100000 person-years, respectively. The greatest absolute reduction was seen in persons $\geq 85$ years (195.9/100 000). During 2014-2015, there were a total of 20506 pneumonia hospitalisations among the 1107240 persons $\geq 65$ years of age; the estimated rate decrease translates to 1456 fewer pneumonia hospitalisations in this age group. Likewise, the estimated rate decreases in age groups 18-49 years (population: 2180 022) and 50-64 years (population: 1117 998) translate to 961 and 1572 fewer pneumonia hospitalisations in 2014-2015, respectively. Persons $<65$ years of age had an estimated $41 \%$ reduction in pneumococcal pneumonia. Similar per cent reduction was seen in persons $\geq 85$ years of age, with the greatest absolute reduction in pneumococcal pneumonia rates (13.0 episodes/100 000). In persons 75-84 years of age, however, the estimated rates were higher than expected by 5.4 episodes/100 000. By the end of the study period, the overall and age-stratified rates of empyema were significantly lower than expected, except for the youngest and the oldest age groups (table 3).

\section{Control condition: trends in all-cause hospitalisations}

Before PCV10 introduction, all-cause hospitalisations (excluding pneumonia) decreased annually by $3.5 \%$ (IRR 0.965 ; 95\% CI 
Table 2 Trends in hospitalisations for all-cause pneumonia, pneumococcal pneumonia and empyema in adults $\geq 18$ years of age before and after introduction of 10-valent pneumococcal conjugate vaccine (PCV10) in the national infant vaccination programme, Finland

\begin{tabular}{|c|c|c|c|c|c|c|c|}
\hline \multirow[b]{4}{*}{ Outcome } & \multirow{4}{*}{$\begin{array}{l}\text { Baseline rate in } 2004-2005 \\
\text { (per } 100000 \text { population) }\end{array}$} & \multicolumn{6}{|c|}{ Adjusted annual trends using interrupted time-series analysis } \\
\hline & & \multicolumn{3}{|c|}{$\begin{array}{l}\text { Period before PCV10 (from 2004-2005 to } \\
\text { 2009-2010*) }\end{array}$} & \multicolumn{3}{|c|}{ Period after PCV10 (from 2011-2012 to 2014-2015*) } \\
\hline & & \multicolumn{3}{|c|}{ Annual trend } & \multicolumn{3}{|c|}{ Annual trend } \\
\hline & & IRR & $95 \% \mathrm{Cl}$ & pt & IRR & $95 \% \mathrm{Cl}$ & pt \\
\hline \multicolumn{8}{|c|}{ All-cause pneumonia } \\
\hline \multicolumn{8}{|c|}{ Age (years) } \\
\hline $18-49$ & 167.9 & 1.012 & 0.988 to 1.037 & 0.075 & 0.908 & 0.876 to 0.953 & $<0.001$ \\
\hline $50-64$ & 310.6 & 1.049 & 1.037 to 1.062 & $<0.001$ & 0.908 & 0.876 to 0.930 & $<0.001$ \\
\hline$\geq 65 \ddagger$ & 1639.8 & 1.018 & 1.013 to 1.023 & $<0.001$ & 0.976 & 0.965 to 0.988 & 0.004 \\
\hline $65-74$ & 816.7 & 1.024 & 1.012 to 1.037 & $<0.001$ & 0.965 & 0.942 to 0.988 & 0.016 \\
\hline $75-84$ & 2071.8 & 1.012 & 1.007 to 1.024 & $<0.001$ & 0.976 & 0.965 to 1.002 & 0.081 \\
\hline$\geq 85$ & 4434.3 & 1.024 & 1.012 to 1.028 & $<0.001$ & 0.988 & 0.965 to 1.012 & 0.375 \\
\hline Total‡ & 502.8 & 1.024 & 1.018 to 1.037 & $<0.001$ & 0.953 & 0.942 to 0.965 & $<0.001$ \\
\hline \multicolumn{8}{|c|}{ Pneumococcal pneumonia } \\
\hline \multicolumn{8}{|c|}{ Age (years) } \\
\hline $18-49$ & 5.7 & 1.037 & 1.002 to 1.087 & 0.029 & 0.855 & 0.775 to 0.953 & 0.003 \\
\hline $50-64$ & 10.4 & 1.062 & 1.024 to 1.087 & 0.001 & 0.855 & 0.785 to 0.930 & $<0.001$ \\
\hline$\geq 65 \ddagger$ & 16.3 & 1.012 & 0.988 to 1.049 & 0.290 & 0.988 & 0.919 to 1.062 & 0.845 \\
\hline $65-74$ & 11.3 & 1.037 & 0.988 to 1.087 & 0.073 & 0.976 & 0.876 to 1.074 & 0.604 \\
\hline $75-84$ & 20.9 & 0.988 & 0.942 to 1.037 & 0.515 & 1.087 & 0.965 to 1.224 & 0.186 \\
\hline$\geq 85$ & 25.6 & 1.024 & 0.953 to 1.087 & 0.590 & 0.865 & 0.711 to 1.037 & 0.105 \\
\hline Totalł & 9.1 & 1.037 & 1.012 to 1.049 & $<0.001$ & 0.919 & 0.876 to 0.965 & $<0.001$ \\
\hline \multicolumn{8}{|l|}{ Empyema } \\
\hline \multicolumn{8}{|l|}{ Age (years) } \\
\hline $18-49$ & 2.4 & 1.024 & 0.976 to 1.062 & 0.378 & 0.976 & 0.865 to 1.114 & 0.736 \\
\hline $50-64$ & 7.4 & 1.049 & 1.012 to 1.087 & 0.015 & 0.930 & 0.844 to 1.024 & 0.117 \\
\hline$\geq 65 \ddagger$ & 7.7 & 1.100 & 1.062 to 1.154 & $<0.001$ & 0.919 & 0.844 to 1.012 & 0.074 \\
\hline $65-74$ & 8.9 & 1.100 & 1.037 to 1.154 & $<0.001$ & 0.930 & 0.824 to 1.049 & 0.248 \\
\hline $75-84$ & 4.7 & 1.114 & 1.049 to 1.196 & 0.001 & 0.908 & 0.766 to 1.074 & 0.266 \\
\hline$\geq 85$ & 11.7 & 1.114 & 1.012 to 1.253 & 0.035 & 0.908 & 0.702 to 1.168 & 0.435 \\
\hline Totalł & 4.8 & 1.062 & 1.037 to 1.087 & $<0.001$ & 0.942 & 0.897 to 1.002 & 0.059 \\
\hline
\end{tabular}

IRRs are adjusted for sex and seasonality, with the natural log of the population size as the offset variable. The IRR of the trend before PCV10 is estimated as the change in annual hospitalisation rates in the years 2004-2005 to 2009-2010. The IRR of the trend after PCV10 is estimated as the comparison of the annual trend in the years 2011-2012 to 2013-2014 to the trend in the period before.

*PCV10 was introduced in the Finnish NVP in September 2010. The year 2010-2011 was defined as a transitional period and was excluded from the analysis. †Two-tailed $p$ Value.

$\ddagger$ Analyses for the aggregate age groups (ie, the total ( $\geq 18$ years of age) and the $\geq 65$ years of age) were age adjusted using the following age groups: all age groups in the analyses for the total and the $65-74,75-84$ and $\geq 85$ years of age in the analyses for the $\geq 65$ years of age group.

IRR, incidence rate ratio; NVP, National Vaccination Programme.

0.953 to 0.976). The trend continued during the PCV10 period (online supplementary figure 1).

\section{DISCUSSION}

This study provides evidence of herd effects and population-level impact of an infant PCV10 programme on adult pneumonia hospitalisations in a high vaccine uptake setting. The analysis of trends and potential outcomes showed an increasing prevaccine trend in adult pneumonia hospitalisations, followed by significant declines in overall and age-stratified rates after PCV10 introduction. By 2014-2015, the rate of all-cause pneumonia hospitalisations in adults had declined by $15 \%$ or 109 episodes per 100000 population. Our data highlight both the substantial burden and opportunities for prevention of pneumonia in the elderly: reductions in all-cause pneumonia hospitalisations in persons $\geq 65$ years of age indicated about 1500 fewer annual hospitalisations.

During the period before PCV10, rates of pneumonia admissions in adults $\geq 18$ years of age increased by $2.4 \%$ annually. Greater rate increases were seen in hospitalisations in which the presumptive aetiology was reported, as the overall rates of pneumococcal pneumonia and empyema increased by $3.7 \%$ and $6 \%$ annually. Increasing trends in adult pneumonia admissions were seen in England during 1997-2005, in Denmark during 1994-2004, and in the USA during 1988-2002 (ie, mostly before introduction of infant PCV7 programmes in these 
Table 3 Estimated and expected hospitalisation rates of all-cause pneumonia in adults $\geq 18$ years of age, 2014-2015

\begin{tabular}{|c|c|c|c|c|c|}
\hline \multirow[b]{2}{*}{ Outcome } & \multirow[b]{2}{*}{$\begin{array}{l}\text { Estimated rate per } \\
100000 \text { population* }\end{array}$} & \multirow[b]{2}{*}{$\begin{array}{l}\text { Expected rate per } 100000 \\
\text { populationt }\end{array}$} & \multicolumn{3}{|c|}{ Expected versus estimated hospitalisation rates in 2014-2015 } \\
\hline & & & $\begin{array}{l}\text { Rate difference per } 100000 \\
\text { population }\end{array}$ & $\begin{array}{l}95 \% \mathrm{Cl} \text { per } 100000 \\
\text { population }\end{array}$ & Per cent reduction (\%) \\
\hline \multicolumn{6}{|c|}{ All-cause pneumonia } \\
\hline \multicolumn{6}{|c|}{ Age (years) } \\
\hline $18-49$ & 120.1 & 164.2 & 44.1 & 35.3 to 52.9 & 26.9 \\
\hline $50-64$ & 348.8 & 488.8 & 140.6 & 122.1 to 158.9 .0 & 28.8 \\
\hline$\geq 65 \S$ & 1820.4 & 1951.9 & 131.5 & 106.0 to 156.9 & 6.7 \\
\hline $65-74$ & 869.3 & 968.4 & 99.1 & 73.1 to 125.0 & 10.2 \\
\hline $75-84$ & 2260.5 & 2417.3 & 156.8 & 106.7 to 206.9 & 6.5 \\
\hline$\geq 85$ & 5097.5 & 5293.3 & 195.9 & 77.3 to 314.4 & 3.7 \\
\hline Total§ & 598.8 & 708.0 & 109.3 & 96.5 to 121.9 & 15.4 \\
\hline \multicolumn{6}{|c|}{ Pneumococcal pneumonia } \\
\hline \multicolumn{6}{|l|}{ Age (years) } \\
\hline $18-49$ & 4.6 & 7.9 & 3.3 & 1.9 to 4.6 & 41.7 \\
\hline $50-64$ & 10.0 & 17.1 & 7.1 & 4.7 to 9.5 & 41.4 \\
\hline$\geq 65 \S$ & 18.9 & 19.4 & 0.5 & -0.9 to 1.9 & 2.5 \\
\hline $65-74$ & 17.4 & 19.1 & 1.7 & -0.7 to 4.2 & 9.1 \\
\hline $75-84$ & 21.7 & 16.3 & -5.4 & -5.9 to -4.8 & -33.0 \\
\hline$\geq 85$ & 18.9 & 31.9 & 13.0 & 3.4 to 22.6 & 40.6 \\
\hline Total§ & 9.5 & 12.9 & 3.5 & 2.5 to 4.4 & 26.5 \\
\hline \multicolumn{6}{|l|}{ Empyema } \\
\hline \multicolumn{6}{|l|}{ Age (years) } \\
\hline $18-49$ & 2.8 & 3.0 & 0.2 & -0.1 to 0.6 & 7.0 \\
\hline $50-64$ & 8.5 & 11.1 & 2.6 & 1.2 to 4.0 & 23.4 \\
\hline$\geq 65 \S$ & 13.8 & 18.4 & 4.6 & 1.9 to 7.2 & 24.9 \\
\hline $65-74$ & 13.4 & 17.3 & 3.8 & 0.5 to 7.1 & 22.2 \\
\hline $75-84$ & 13.8 & 19.1 & 5.3 & 0.2 to 10.4 & 27.8 \\
\hline$\geq 85$ & 15.9 & 22.5 & 6.6 & -3.3 to 16.4 & 29.2 \\
\hline Total§ & 7.0 & 8.5 & 1.5 & 0.9 to 2.1 & 17.5 \\
\hline
\end{tabular}

*Estimated rate is the average hospitalisation rate estimated from the adjusted negative binomial regression model, which included as independent variables the time since the beginning of the study and time since the start of the intervention period. The year 2010-2011 was considered a transitional period and excluded from the analysis. tExpected rate is the average hospitalisation rate estimated from the adjusted model with the time since the beginning of the study as the independent variable. $\$ 95 \% \mathrm{Cls}$ are estimated using Delta method and were considered significant if they did not include zero.

$\S$ Analyses for the aggregate age groups (ie, the total ( $\geq 18$ years of age) and the $\geq 65$ years of age) were age adjusted using the following age groups: all age groups in the analyses for the total, and the $65-74,75-84$ and $\geq 85$ years of age in the analyses for the $\geq 65$ years of age group.

countries). ${ }^{23-25}$ The long-term increasing trend in pneumonia hospitalisations may be associated with ageing of the population and increase in the prevalence of underlying medical conditions, both recognised risk factors for CAP. ${ }^{26}$ The proportion of elderly hospitalised pneumonia patients who had underlying medical conditions increased in the USA during $1988-2002 .{ }^{25}$ In the UK, however, an increase in pneumonia admissions in the elderly during 1998-2010 was independent of increases in comorbidities and was postulated to be associated with changes in service provision and health-seeking behaviour. ${ }^{27}$ From 1996 to 2009, the number of pneumonia hospitalisations in Finland increased by $42 \%$, primarily in the elderly; the duration of hospital stay, however, decreased. ${ }^{28}$ Changes in coding practices may also be associated with increases in pneumonia hospitalisations, but this is unlikely to have influenced our findings because we used only the primary discharge diagnosis and included all pneumonia-related ICD-10 codes in the analysis. We conducted an additional analysis of all episodes in which ICD-10-coded pneumonia was listed in any position of the discharge diagnoses, with or without hospitalisation. The relative reductions in pneumonia were similar to the analysis in which the case definition was restricted to the primary discharge diagnosis, but the absolute reductions were larger (online supplementary tables 1 and 2).

Our trend analysis showed significant declines in rates of all-cause pneumonia hospitalisations following PCV10 introduction. In persons $\geq 65$ years of age, the estimated annual decline was $2.4 \%$, and in those 18-49 and 50-64 years of age, it was $9.2 \%$. In younger adults, rates of pneumococcal pneumonia also decreased. The large burden of all-cause pneumonia hospitalisations in persons $\geq 85$ years of age, however, was associated with the greatest absolute reduction, an estimated 195.9 fewer episodes per 100000 than expected, that is, had the increasing pre-PCV10 trend continued. Population-based prospective surveillance studies have consistently reported that rates of CAP hospitalisations increase with advancing age. ${ }^{25}$ In Finland, pneumonia hospitalisations were previously projected to increase by 49\% from 2010 to $2030 .^{28}$ Percentage reductions in all-cause pneumonia hospitalisations, however, were greatest in young 


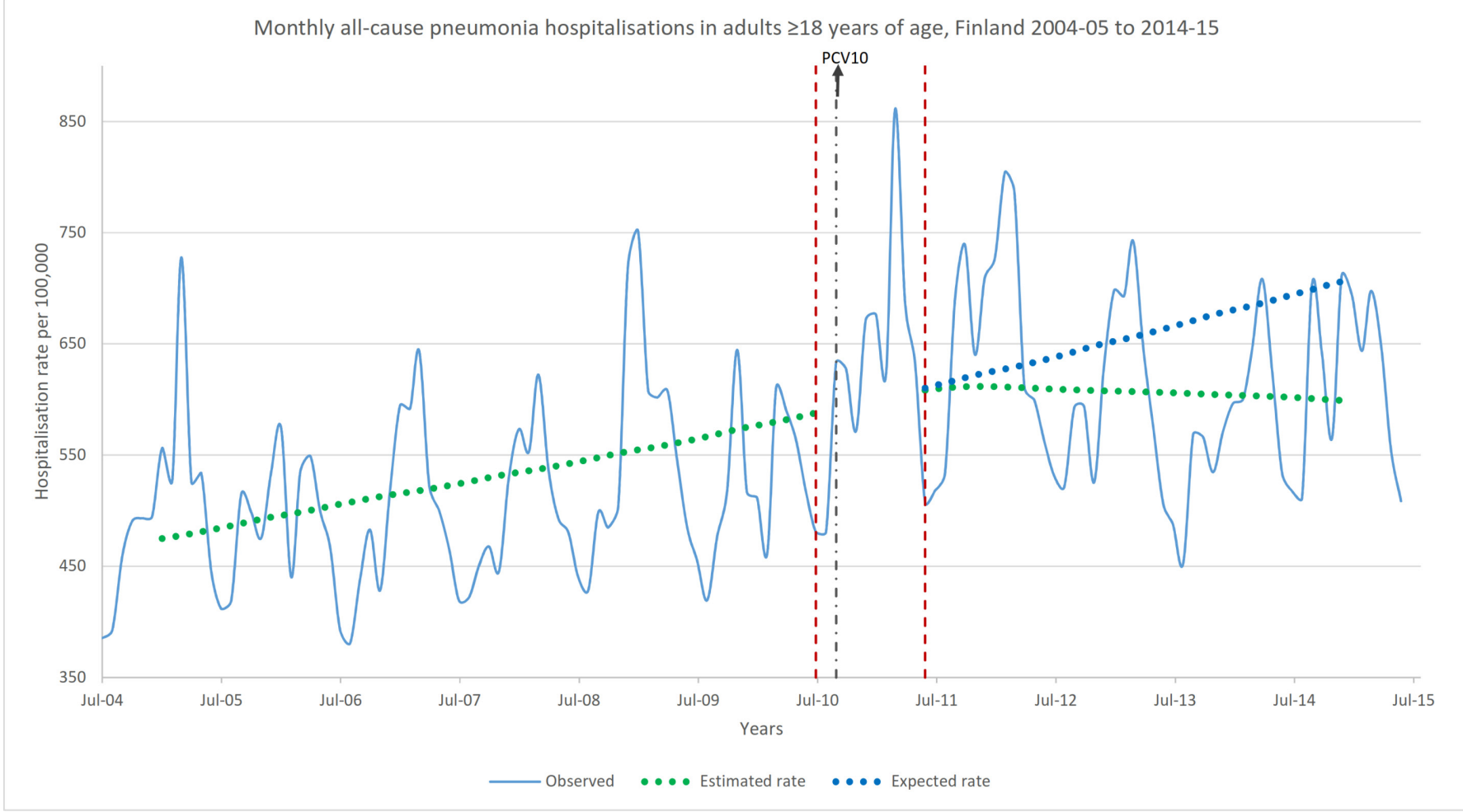

Figure 1 The vertical black dash-dot line marks to the introduction of PCV10 in the Finnish National Vaccination Programme. The period between the vertical red dash lines, that is, July 2010-June 2011, is a transitional period and was excluded from the analysis. Observed rate is the unadjusted average monthly hospitalisation rate. Estimated rate is the average hospitalisation rate estimated from the adjusted negative binomial regression model with the full set of parameters (ie, time since the beginning of the study and time since the start of the intervention period). Expected rate is the average rate estimated from the model with time since the beginning of the study as the only independent variable. Twelve-month moving average filter was applied to both estimated and expected rates. PCV10, 10-valent pneumococcal conjugate vaccine.

adults $(26.9 \%$ in adults $18-49$ years of age), which might be associated with reduced exposure to PCV10 serotypes in parents of vaccinated children. Although previous studies evaluating the impact of infant PCV7 programmes on adult pneumonia hospitalisations have consistently showed declines in pneumococcal pneumonia, the reported changes in age-specific all-cause pneumonia hospitalisation rates have varied across settings. ${ }^{15} 17$ This variation could be associated with several factors or their combination, including differences in infant vaccination programmes (eg, uptake, schedule or catch-up), population characteristics, coding practices, admission criteria or analytical methods.

Some limitations should be considered when interpreting the findings. First, the study design was ecological. Although our study aimed to estimate the indirect effect of infant PCV10, other adult vaccines, such as influenza, PCV13 and the PPSV23, may also decrease the risk of pneumonia hospitalisation. In Finland, influenza vaccination has been recommended for all persons $\geq 65$ years of age since 2002 . During our study period, the annual vaccine coverage varied from $38 \%$ to $50 \%$. Uptake was highest during the 2009 influenza pandemic but actually decreased in subsequent years, that is, during PCV10 period. In 2014, the cumulative coverage of PPSV23 and PCV13 in adults was $<5 \%$ based on vaccine distribution data. This low coverage would not be expected to have a population-level impact on overall pneumonia incidence.

Second, all-cause pneumonia is a non-specific outcome, and trends in other respiratory pathogens may influence its occurrence. In 2010-2011, an increase in all-cause pneumonia was observed. Although this coincided with an epidemic of $\mathrm{Myco-}$ plasma pneumoniae infections in Finland, it is unlikely to have influenced our findings as most cases were in younger age groups (5-19 years of age), and the period (late-2010 to mid-2011) was excluded from our analysis. ${ }^{29}$ In our study, some $95 \%$ of episodes were recorded as pneumonia due to unspecified cause, indicating the lack of sensitive and specific aetiological diagnosis for pneumonia in a routine hospital care. ${ }^{34}$ To capture all episodes of pneumococcal pneumonia and empyema, we used broader case definition (with or without overnight hospitalisations) and also included non-primary discharge diagnoses. Nevertheless, these outcomes accounted only for $<2 \%$ of pneumonia episodes in our study. In previous reports, laboratory-confirmed pneumococcal pneumonia accounted for a small fraction of all-cause pneumonia hospitalisations. ${ }^{15-17}$ In addition, our sensitivity analysis showed no significant changes in pneumonia hospitalisations due to specified pathogens other than S. pneumoniae (data not shown). Our case definition also included healthcare-associated pneumonia, which is difficult to distinguish from CAP in hospital discharge records and is mainly caused by bacteria other than $S$. pneumoniae.

Third, administrative data are subject to misclassification, secular changes in coding and clinical practices, as well as criteria for admission. ${ }^{30}$ However, an Australian validation study estimated 98\% sensitivity, 97\% specificity, 96\% positive predictive value and 98\% negative predictive value for ICD-10-coded pneumonia hospitalisations. ${ }^{31}$ In the USA, comparison of IPD rates reported by active surveillance with those estimated using ICD-coded data showed similar temporal trends. ${ }^{10}$ Changes in admission criteria might be associated with the observed changes in pneumonia hospitalisation rates, particularly if there was a shift to management of less severe cases in outpatient settings. ${ }^{32}$ 
Monthly all-cause pneumonia hospitalizations in adults by age group, Finland 2004-05 to 2014-15
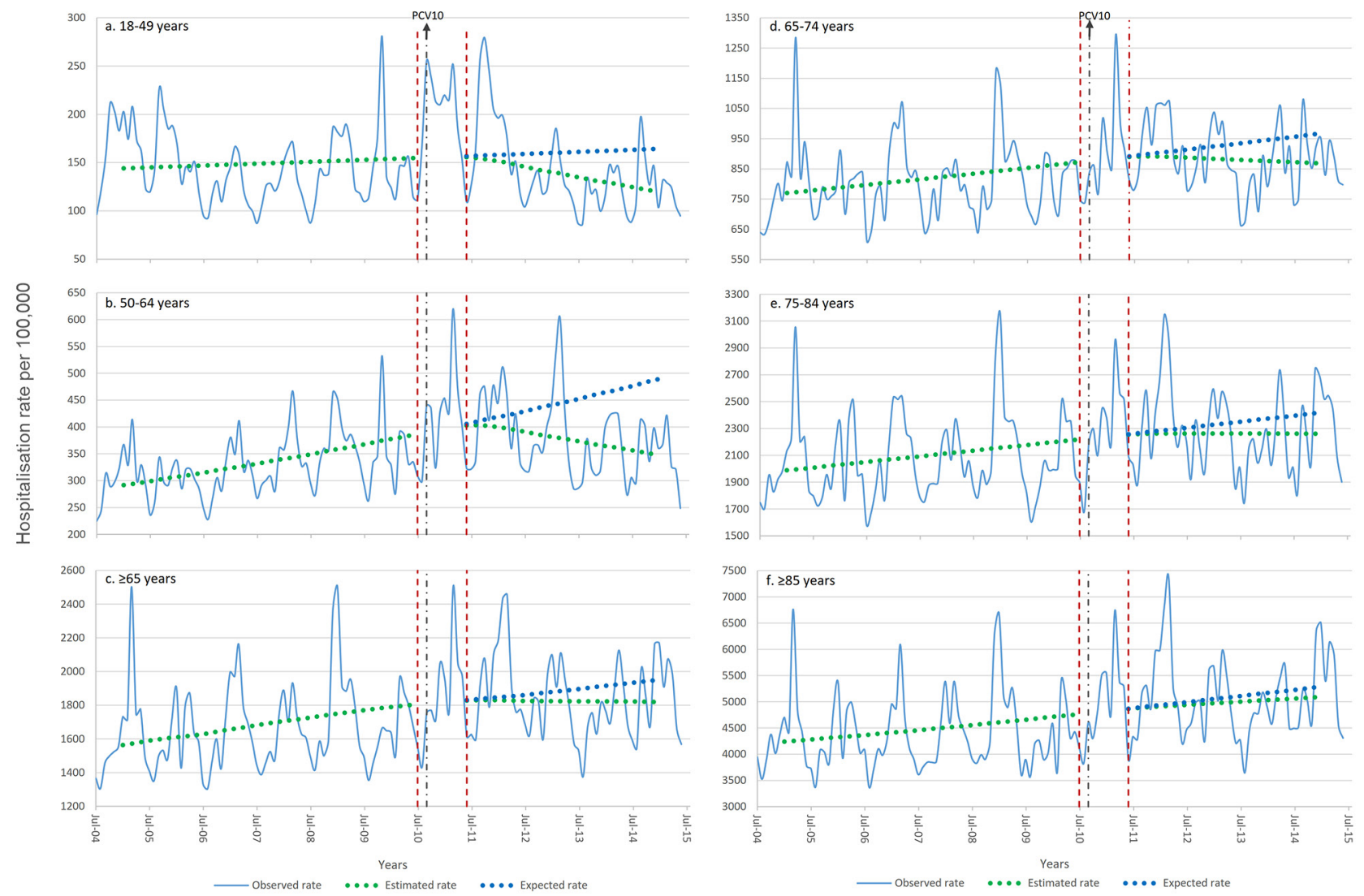

Figure 2 The vertical black dash-dot line marks to the introduction of PCV10 in the Finnish National Vaccination Programme. The period between the vertical red dash lines, that is, July 2010-June 2011, is a transitional period and was excluded from the analysis. Observed rate is the unadjusted average monthly hospitalisation rate. Estimated rate is the average hospitalisation rate estimated from the adjusted negative binomial regression model with the full set of parameters (ie, time since the beginning of the study and time since the start of the intervention period). Expected rate is the average rate estimated from the model with time since the beginning of the study as the only independent variable. Twelve-month moving average filter was applied to both estimated and expected rates. PVC10, 10-valent pneumococcal conjugate vaccine.

The Finnish guidelines for the management of CAP published in 2008 introduced criteria for identifying low-risk patients who could be managed on outpatient basis. ${ }^{33}$ We therefore conducted a sensitivity analysis that showed that pneumonia hospitalisations continued to increase after the guidelines were published and began to decrease only during the PCV10 period (online supplementary figure 2). However, trends in outpatient visits for CAP could not be examined, as the outpatient register was established only after PCV10 introduction. The results of an additional analysis, which also included outpatient and emergency room pneumonia episodes, were similar to the analysis restricted to hospitalised episodes (online supplementary tables 1 and 2). It is possible that the decreasing trend in hospitalisations other than pneumonia might be associated with a shift towards outpatient management of various conditions, but this is unlikely to have influenced the observed trends in all-cause pneumonia hospitalisations.

Last, increases in chronic medical conditions that increase the risk of pneumococcal pneumonia also contribute to the disease burden. We could not assess the potential effect of underlying conditions in this study due to the complexity of obtaining comprehensive data on these conditions. Assessing whether indirect vaccine effects against pneumonia are different in persons with and without comorbidities will help better defining the characteristics of herd protection in adults.

The strengths of this study included the use of comprehensive, nationwide, population-based register data with a case definition for pneumonia hospitalisation with increased specificity of the main outcome, hospital-treated primary pneumonia. Although our dataset did not include radiological data, it is likely that our case definition captured most radiologically confirmed episodes as patients were hospitalised at least overnight, and pneumonia was the primary discharge diagnosis. Our data enabled using interrupted time-series analysis with sufficient data points before and after infant PCV10 to estimate the herd effect on adult pneumonia hospitalisations. This analysis method had advantages over the two-point, beforeafter design because it incorporated multiple time points and enabled accounting for seasonal variation and, importantly, the prevaccine secular increase in pneumonia hospitalisations in Finland. ${ }^{28}$ Last, our analysis showed the trends in all-cause hospitalisations or potential shifts in outpatient management of CAP were unlikely to explain the trends in pneumonia hospitalisations.

In conclusion, these national data suggest that herd protection from infant PCV10 has reversed the increasing trend and 
substantially decreased all-cause pneumonia hospitalisations in adults, particularly in the elderly. This finding is significant as the number of persons $\geq 65$ years of age in Finland is projected to increase by $17 \%$ from 2014 to 2020 , likely increasing the burden of CAP hospitalisations even further. ${ }^{34}$ The findings also have major implications for economic analyses comparing strategies for prevention of pneumococcal diseases in adults.

Acknowledgements We would like to thank Dr Hanna Nohynek for her comments on an earlier version of this manuscript. 00 was supported by the International Doctoral Programme in Epidemiology, Health Sciences, Faculty of Social Sciences, University of Tampere, Finland.

Contributors Study concept and design: 00, AAP, JJ and JPN. Acquisition of data: HR-K and ER. Analysis and interpretation of data: OO, HR-K, AAP, JJ and JPN. Drafting of the manuscript: 00 and JPN. Critical revision of the manuscript for important intellectual content: 00, HR-K, AAP, JJ and JPN. Statistical analysis: 00 and HR-K. Obtained funding: JPN. Study supervision: JPN. Final approval: OO, HR-K, $A A P, E R$, JJ and JPN.

Funding The study was funded by The National Institute for Health and Welfare (THL), and Health Sciences, Faculty of Social Sciences, University of Tampere, Finland. The study was partially supported by the Integrated Monitoring of Vaccine in Europe (I-MOVE+) project, which has received funding from the European Union's Horizon 2020 research and innovation programme under grant agreement no. 634446.

Competing interests 00 and JPN: no conflict of interest. HR-K, ER, AAP, and JJ are employees of the National Institute for Health and Welfare, Department of Public Health Solutions, which has received research funding from GlaxoSmithKline for a nationwide effectiveness trial of the 10-valent pneumococcal conjugate vaccine. HR$\mathrm{K}, \mathrm{AAP}, \mathrm{ER}$, and JJ are coinvestigators in the trial.

Ethics approval National Institute for Health and Welfare (THL), Helsinki. Provenance and peer review Not commissioned; externally peer reviewed. (c) Article author(s) (or their employer(s) unless otherwise stated in the text of the article) 2018. All rights reserved. No commercial use is permitted unless otherwise expressly granted.

\section{REFERENCES}

1 Wang H, Naghavi M, Allen C, et al. Global, regional, and national life expectancy, all-cause mortality, and cause-specific mortality for 249 causes of death, 19802015: a systematic analysis for the Global Burden of Disease Study 2015. Lancet 2016;388:1459-544

2 Welte T, Torres A, Nathwani D. Clinical and economic burden of community-acquired pneumonia among adults in Europe. Thorax 2012;67:71-9.

3 Said MA, Johnson HL, Nonyane BA, et al. Estimating the burden of pneumococcal pneumonia among adults: a systematic review and meta-analysis of diagnostic techniques. PLoS One 2013;8:e60273.

4 Rozenbaum MH, Pechlivanoglou P, van der Werf TS, et al. The role of Streptococcus pneumoniae in community-acquired pneumonia among adults in Europe: a metaanalysis. Eur J Clin Microbiol Infect Dis 2013:32:305-16.

5 Palmu AA, Saukkoriipi A, Snellman M, et al. Incidence and etiology of communityacquired pneumonia in the elderly in a prospective population-based study. Scand J Infect Dis 2014;46:250-9.

6 Jain S, Self WH, Wunderink RG, et al. Community-acquired pneumonia requiring hospitalization among U.S. adults. N Eng/ J Med 2015;373:415-27.

7 Black SB, Shinefield HR, Ling S, et al. Effectiveness of heptavalent pneumococcal conjugate vaccine in children younger than five years of age for prevention of pneumonia. Pediatr Infect Dis J 2002;21:810-5.

8 Cutts FT, Zaman SM, Enwere G, et al. Efficacy of nine-valent pneumococcal conjugate vaccine against pneumonia and invasive pneumococcal disease in The Gambia: randomised, double-blind, placebo-controlled trial. Lancet 2005;365:1139-46.

9 Bonten MJ, Huijts SM, Bolkenbaas M, et al. Polysaccharide conjugate vaccine against pneumococcal pneumonia in adults. N Engl J Med 2015;372:1114-25.
10 Simonsen L, Taylor RJ, Young-Xu Y, et al. Impact of pneumococcal conjugate vaccination of infants on pneumonia and influenza hospitalization and mortality in all age groups in the United States. MBio 2011;2:e00309-10.

11 Saxena S, Atchison C, Cecil E, et al. Additive impact of pneumococcal conjugate vaccines on pneumonia and empyema hospital admissions in England. I Infect 2015;71:428-36.

12 Palmu AA, Rinta-Kokko H, Nohynek H, et al. Impact of ten-valent pneumococcal conjugate vaccine on pneumonia in Finnish children in a nation-wide populationbased study. PLoS One 2017;12:e0172690.

13 van Hoek AJ, Sheppard CL, Andrews NJ, et al. Pneumococcal carriage in children and adults two years after introduction of the thirteen valent pneumococcal conjugate vaccine in England. Vaccine 2014;32:4349-55.

14 Grijalva CG, Nuorti JP, Arbogast PG, et al. Decline in pneumonia admissions after routine childhood immunisation with pneumococcal conjugate vaccine in the USA: a time-series analysis. Lancet 2007:369:1179-86.

15 Griffin MR, Zhu Y, Moore MR, et al. U.S. hospitalizations for pneumonia after a decade of pneumococcal vaccination. N Engl J Med 2013:369:155-63.

16 Jardine A, Menzies RI, McIntyre PB. Reduction in hospitalizations for pneumonia associated with the introduction of a pneumococcal conjugate vaccination schedule without a booster dose in Australia. Pediatr Infect Dis / 2010;29:607-12.

17 Menzies RI, Jardine A, Mclntyre PB. Pneumonia in elderly australians: reduction in presumptive pneumococcal hospitalizations but No change in all-Cause pneumonia hospitalizations following 7-valent pneumococcal conjugate vaccination. Clin Infect Dis 2015:61:927-33.

18 Nuorti P, Reingold A. Preventing pneumococcal infections in older adults. Lancet Respir Med 2015:3:834-6

19 National Institute for Health and Welfare (THL). Rokotusrekisteri (The Vaccination Register). https://www.thl.fi/fi/web/rokottaminen/kansallinen-rokotusohjelma/ rokotusrekisteri (accessed 22 Jun 2017).

20 Hilbe J. Negative Binomial Regression. 2nd edn. Cambridge: Cambridge University Press, 2011.

21 Feikin DR, Kagucia EW, Loo JD, et al. Serotype Replacement Study Group. Serotypespecific changes in invasive pneumococcal disease after pneumococcal conjugate vaccine introduction: a pooled analysis of multiple surveillance sites. PLOS Med 2013:10:e1001517

22 Wagner AK, Soumerai SB, Zhang F, et al. Segmented regression analysis of interrupted time series studies in medication use research. J Clin Pharm Ther 2002;27:299-309.

23 Trotter CL, Stuart JM, George R, et al. Increasing hospital admissions for pneumonia, England. Emerg Infect Dis 2008;14:727-33.

24 Thomsen RW, Riis A, Nørgaard M, et al. Rising incidence and persistently high mortality of hospitalized pneumonia: a 10-year population-based study in Denmark. J Intern Med 2006;259:410-7.

25 Fry AM, Shay DK, Holman RC, et al. Trends in hospitalizations for pneumonia among persons aged 65 years or older in the United States, 1988-2002. JAMA 2005;294:2712-9.

26 Torres A, Peetermans WE, Viegi G, et al. Risk factors for community-acquired pneumonia in adults in Europe: a literature review. Thorax 2013;68:1057-65.

27 Millett ER, De Stavola BL, Quint JK, et al. Risk factors for hospital admission in the 28 days following a community-acquired pneumonia diagnosis in older adults, and their contribution to increasing hospitalisation rates over time: a cohort study. BMJ Open 2015;5:e008737

28 Koskela H. Current and future needs for hospital treatment of pneumonia [Article in Finnish]. Suomen Lääkärilehti 2013;68:1349-55.

29 Polkowska A, Harjunpää A, Toikkanen S, et al. Increased incidence of Mycoplasma pneumoniae infection in Finland, 2010-2011. Euro Surveill 2012:17:20072.

30 Manderbacka K, Arffman M, Lyytikäinen 0 , et al. What really happened with pneumonia mortality in Finland in 2000-2008?: a cohort study. Epidemiol Infect 2013;141:800-4

31 Skull SA, Andrews RM, Byrnes GB, et al. ICD-10 codes are a valid tool for identification of pneumonia in hospitalized patients aged $>$ or $=65$ years. Epidemiol Infect 2008;136:232-40.

32 Schuck-Paim C, Taylor RJ, Simonsen L, et al. Challenges to estimating vaccine impact using hospitalization data. Vaccine 2017;35:118-24.

33 Current care guidelines - pneumonia [Article in Finnish]. The Finnish Medical Society. Duodecim 2008;124:2030-9. Available online at: www.kaypahoito.fi.

34 Statistics Finland. Official Statistics of Finland (OSF): population projection 2012 2060. http://www.stat.fi/til/vaenn/2012/vaenn_2012_2012-09-28_tie_001_en.html (accessed 20 Feb 2016). 